\title{
Recent Trends in Solid-Phase Extraction for Environmental, Food and Biological Sample Preparation
}

\author{
Anna Laura Capriotti ${ }^{1}$. Giorgia La Barbera ${ }^{1,2}$. Susy Piovesana ${ }^{1}$
}

Published online: 16 July 2019

๑) Springer-Verlag GmbH Germany, part of Springer Nature 2019

Analytical chemistry is fundamental not only for routine analysis, but also for important multidisciplinary fields, such as proteomics, food analysis, lipidomics, metabolomics and biomedical applications, just to mention a few. The development of such research areas has been possible due to the enormous improvement in separation techniques and instrumentation, which have allowed the development of methods with improved sensitivity, dynamic range and number of analytes which can be monitored at the same time. However, sometimes the above-mentioned improvements are not sufficient to solve complex analytical problems, and sample preparation, which allows the clean-up and enrichment of target analytes, frequently remains a critical step in most analytical methods.

Among the different strategies which can be pursued to separate the target analytes, solid-phase extraction (SPE) is the most widely used method for the extraction, clean-up, concentration, and fractionation of analytes in environmental, food and biological samples. Today, the demand for highly efficient, selective, sensitive, reproducible and green analytical methodologies for complex sample preparation, fractionation and/or extraction of analytes, requires continuous advances in separation media and techniques.

For this reason, the Topical Collection "Recent trends in solid-phase extraction for environmental, food and biological sample preparation" is dedicated to the most recent descriptions of SPE in the literature. The Topical Collection comprises seven review articles and three original papers. The review articles

Published in the topical collection Recent Trends in Solid-Phase Extraction for Environmental, Food and Biological Sample

Preparation with guest editors Anna Laura Capriotti, Giorgia La Barbera, and Susy Piovesana.

Susy Piovesana

susy.piovesana@uniroma1.it

1 Department of Chemistry, Università di Roma "La Sapienza", Piazzale Aldo Moro 5, 00185 Rome, Italy

2 Department of Nutrition, Exercise and Sports, University of Copenhagen, Nørre Allé 51, 2200 Copenhagen, Denmark provide a snapshot of the current state of SPE for the investigation of pollutants in environmental analysis, also at trace level, food analysis, emerging materials and magnetic materials developed for SPE. The original papers represent recent applications of SPE in the most common form of development of new materials and systems for enrichment of target analytes.

In this context, we would like to thank all the authors who contributed to the success of this Topical Collection for their excellent work. We would like to conclude this introduction to the Topical Collection with the hope that the papers collected here will provide insight and inspiration in the readers for further improvement of this fundamental approach, which is SPE in all its varieties.

Finally, we would like to thank the Editors of Chromatographia for the kind invitation to serve as the guest editor and co-guest editors of this Topical Collection.

\section{Guest Editor}

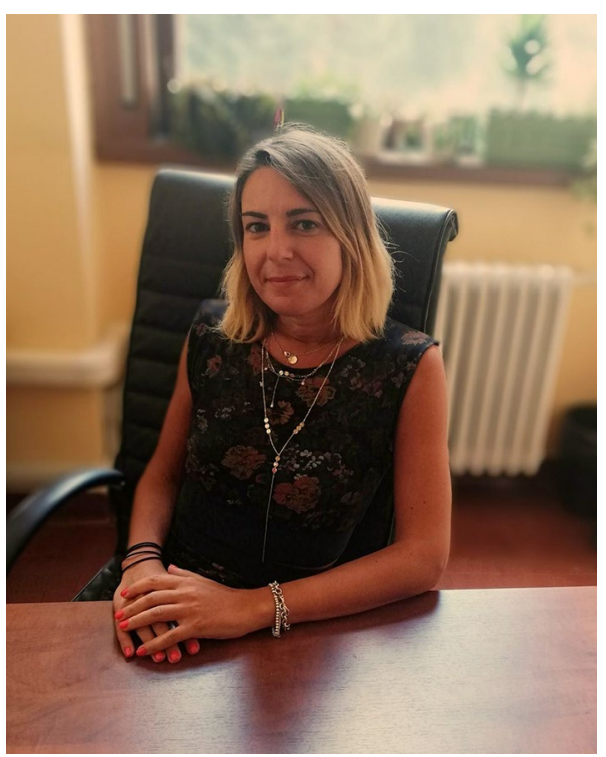

Anna Laura Capriotti is an Associate Professor since October 2018 at the University of Rome "La Sapienza", 
where she received her $\mathrm{PhD}$ in 2012. Her running research lines are mainly focused on the development of analytical platform based on UHPLC and high resolution mass spectrometry in the omic science, in particular peptidomics, phosphoproteomics and lipidomics. She is the principal investigator of different grants. She has been awarded the EuChemS Lecture Award 2017 and she is included in the Power List 2018: Top 40 Under 40 as one of the best young researchers in analytical science. She is author in over 110 papers in high ranked journals.

\section{Co-guest Editors}

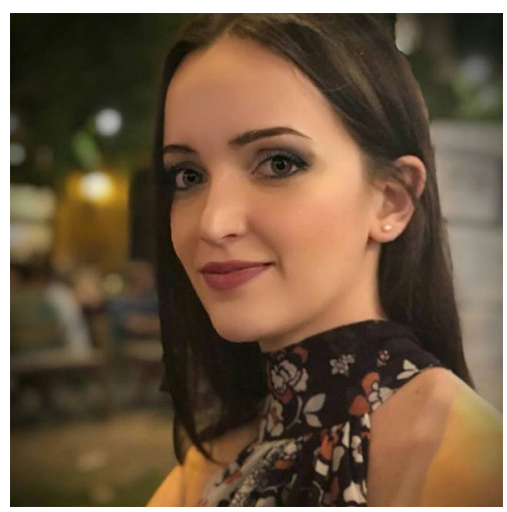

Giorgia La Barbera obtained her PhD in Chemistry in 2017 at the University of Rome "La Sapienza" where she worked as a post doc researcher until May 2019. Currently, she is a post doc researcher at the Department of Nutrition, Exercise and Sport of the University of Copenhagen. During her academic career she worked on several research fields mainly dealing with the development of metabolomics and lipidomics approaches by liquid chromatography coupled to high resolution mass spectrometry. She won several grants either as participant or as principal investigator, among which a Marie Curie Individual Fellowship grant in 2019. Her scientific work led to the publication of 36 scientific papers in high ranked journals. She attended several international conferences with 9 oral communications and 21 poster communications.

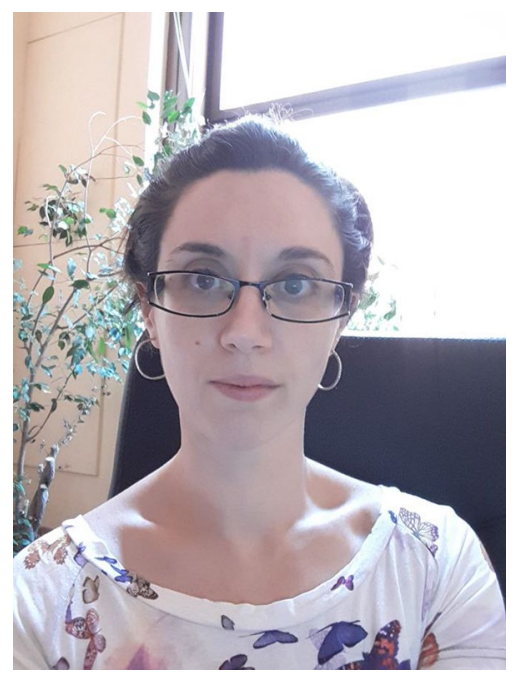

Susy Piovesana is currently working as researcher at the Department of Chemistry of Università di Roma "La Sapienza" (Italy), from where she received her PhD in 2014. Her research interests include the development of analytical methods for UHPLC coupled to mass spectrometry analysis of a variety of analytes, from small molecule target analysis to omics investigations by untargeted analysis. The main topic of her research is proteomics, from global proteome analysis to the specific issues of isolation and enrichment of post-translational modifications. She is co-author of 62 papers on ranked scientific journals.

Publisher's Note Springer Nature remains neutral with regard to jurisdictional claims in published maps and institutional affiliations. 